\title{
5. Die Psychotherapie in der Facharztausbildung
}

\author{
Christoph Schade, Annegret Dreher und Claudia Dambacher
}

\section{Zwischen Couch und Wirklichkeit}

Sie haben sich für die Aus- und Weiterbildung zum Facharzt für Psychiatrie und Psychotherapie entschieden. Dies ist einer der wenigen Facharzttitel, der zwei Qualifikationen beinhaltet. Man stelle sich nun einmal vor, es gäbe einen Facharzt für Innere Medizin und Chirurgie oder Augenheilkunde und Dermatologie. Diese Facharztkombinationen würden schon allein durch die Fülle der Fachinhalte eher abschreckend wirken. Nun haben Sie sich sogar für eine Facharztausbildung entschieden, die auch noch eine Qualifikation beinhaltet, die den „normalen“ Bereich der Schulmedizin verlässt. Warum eigentlich? Reicht Ihnen die Somatik nicht schon aus? Wir gehen mal davon aus, dass genau diese Kombination den Reiz für Sie ausmacht.

Durch diese Komplexität ergeben sich aber auch erhebliche Probleme in dieser Facharztausbildung, die ein Ungleichgewicht der Ausbildungsinhalte zur Folge haben können. Die Facharztausbildung zum Psychiater und Psychotherapeuten findet überwiegend in psychiatrischen Fachabteilungen von Allgemeinkrankenhäusern oder psychiatrischen Fachkrankenhäusern statt. Diese Kliniken sind meist mit dem Versorgungsauftrag für bestimmte Regionen beliehen. Entsprechend stehen häufig akutpsychiatrische Krankheitsbilder im Vordergrund der Behandlung, nicht diejenigen, die Sie eventuell im Rahmen einer eigentlichen Psychotherapie kennenlernen würden. Die Kliniken wählen unterschiedliche Ansätze in der Behandlung. Überwiegend wird ein störungsspezifisches Konzept verfolgt, einige Kliniken favorisieren jedoch eine größere Durchmischung von 
Störungsbildern und Therapieverfahren, sodass schnell der Überblick über die verschiedenen Möglichkeiten ihrer Ausrichtung und Spezialisierung verloren gehen kann oder gar nicht erst entsteht. Damit Sie jedoch nicht völlig den Mut und das Interesse verlieren, wollen wir Ihnen in den nächsten Zeilen auf der einen Seite einen kleinen Überblick geben sowie auf der anderen Seite mögliche Perspektiven aufzeigen.

\section{Die Patienten und ihre Störungsbilder}

Der Weg der Patienten in die Klinik erfolgt zum größten Teil über Notaufnahmen oder nach fachärztlicher Einweisung. Meist handelt es sich um akute Zustandsbilder, wie Exazerbationen von Störungen aus dem schizophrenen Formenkreis oder affektive Erkrankungen, delirante Zustandsbilder, Intoxikationen im Rahmen von Suchterkrankungen oder suizidale Handlungen sowie Krisen bei Persönlichkeitsstörungen. Im Gegensatz zu psychosomatischen bzw. rehabilitativen Einrichtungen werden die meisten Patienten nicht elektiv aufgenommen, sondern in Notfallsituationen. Das bedeutet: Machen Sie sich auf stressige, aber auch interessante Situationen gefasst!

\section{Die Facharztausbildung - eine Bestandsaufnahme}

Die Facharztausbildung umfasst schwerpunktmäßig den psychiatrischen bzw. den biologischen Ansatz. Dies hat zur Folge, dass trotz der formalen Bezeichnung des Facharzttitels „Psychiatrie und Psychotherapie“ ein deutliches Übergewicht in der Vermittlung psychiatrisch-biologischen Wissens liegt und die psychotherapeutischen Behandlungsmöglichkeiten häufig unzureichend vermittelt werden.

In der Psychotherapieausbildung gehen die Weiterbildungsbeauftragten der Kliniken unterschiedliche Wege. Einige bieten die Psychotherapieausbildung in der eigenen Abteilung an; dabei werden die Ausbildungsinhalte häufig von Oberärzten oder psychologischen Mitarbeitern vermittelt. Der praktische Teil wird dann in der eigenen Fachabteilung unter Bedingungen angeboten, die im Grunde nicht denen einer Richtlinienpsychotherapie entsprechen. Andere Kliniken gehen hingegen Kooperationen mit Psychotherapieinstituten ein, die eher auf die Ausbildung von psychologischen Psychotherapeuten und den ambulanten Bereich ausgerichtet sind. Entsprechend erfolgt die Vermittlung der theoretischen Inhalte und die Durchführung der praktischen Therapiestunden außerhalb der Kliniken an den jeweiligen Instituten. Beide Konzepte haben daher Vor- und Nachteile.

Findet beispielsweise die psychotherapeutische Aus- und Weiterbildung in der eigenen Abteilung statt, steht die Versorgung von Akutpatienten im Vordergrund. Die psychotherapeutischen Behandlungen von Angsterkrankungen, Essstörungen, Posttraumatischen Belastungsstörungen sowie Persönlichkeitsstörungen können in diesem Setting nicht in erforderlichem Umfang gewährleistet werden. Allenfalls kann beispielsweise eine komorbide depressive Störung im Sinne einer Krisenintervention bzw. stationären Behandlung adäquat behandelt werden. Zudem besteht für die oben angeführten Störungsbilder häufig keine 
klare akut-psychiatrische bzw. stationäre Behandlungsindikation. Vereinfacht formuliert: Diese Patienten kommen nicht auf eine Akutstation oder bleiben nicht so lange, bis eine adäquate Störungsdiagnostik (beispielsweise einer zugrunde liegenden Persönlichkeitsstörung) oder eine „Psychotherapie“ im eigentlichen Sinne beginnen könnte. Allenfalls können im stationären Rahmen eine basale Diagnostik erfolgen und Hypothesen bzgl. zugrunde liegender weiterer Störungsbilder angestellt werden. Hier bietet ein mehrwöchiger stationär-psychiatrischer Aufenthalt wiederum gute Möglichkeiten zur längerfristigen Verhaltens- oder beispielsweise Interaktionsbeobachtung.

Fazit: In der Klinik findet wenig Psychotherapie statt!

Bei einer Verlagerung der psychotherapeutischen Ausbildung in Ausbildungsinstitute außerhalb der Klinik steht nun wiederum die Versorgung von Akutpatienten, wie sie sich in der Klinik befinden, nicht im Vordergrund, sondern eine längerfristige ambulante Behandlung mittels eines Richtlinienverfahrens. So werden die Behandlungen anders gelagerter Störungsbilder und der Einsatz anderer psychotherapeutischer Methoden ermöglicht. Die ambulante Behandlung neben der Kliniktätigkeit bedeutet eine Menge Vor- und Nachbereitungszeit sowie einen stetigen Perspektivenwechsel.

Fazit: Ambulant lässt tiefer blicken, bedeutet jedoch auch mehr Arbeit!

So stehen Sie in der Weiterbildung vor zwei wesentlichen Entscheidungen: Welches anerkannte Psychotherapieverfahren ist zu wählen (beispielsweise Verhaltenstherapie oder tiefenpsychologisch-fundierte Psychotherapie) und - mit Hinblick auf Ihre weitere ärztliche Tätigkeit - in welchem Umfang widmen Sie sich dem psychotherapeutischen Teil der Ausbildung und in welcher Klinik werden Sie diesbezüglich am besten unterstützt?

Nach initialem Boom und großen therapeutischen Hoffnungen, die sich mit der Entwicklung der Psychopharmaka verbanden (nicht zuletzt gefördert durch die Pharmaindustrie), hat sich in den vergangenen Jahren gezeigt, dass viele dieser Erwartungen nicht erfüllt werden konnten. Die wirklich spannenden Entwicklungen gab es hingegen bei vielen Störungsbildern in neuen psychotherapeutischen Verfahren.

\section{Wie kann Ihre Ausbildung diesen Entwicklungen gerecht werden?}

Die Vorgaben der Ärztekammern sind hier wenig hilfreich. Die Zusammenstellung der geforderten psychotherapeutischen Ausbildungsinhalte wirkt recht konstruiert und nicht bedarfsgerecht. So hat die Gruppen- und Einzelselbsterfahrung ein deutliches Übergewicht, die Vermittlung von theoretischen Ausbildungsinhalten und praktischen Erfahrungen durch die Ableistung von Therapiestunden steht dafür aus nicht nachvollziehbaren Gründen im Hintergrund.

Im Idealfall sollte die psychotherapeutische Ausbildung die Behandlung von akut psychisch kranken Menschen im stationären oder teilstationären Setting, aber auch die weiterführende Versorgung im ambulanten Rahmen berücksichtigen.

Sie und der Patient können davon profitieren, wenn sich die Behandlung nicht nur auf eine Phase der Krankheit beschränkt, sondern der Patient durch einen 
Therapeuten von der akuten Krankheitsphase bis zur ambulanten Weiterbehandlung begleitet wird. Dies bietet die Möglichkeit, die unterschiedlichen Stadien der Erkrankung zu und die jeweils erforderlichen Interventionen in Anlehnung an das bio-psycho-sozialen Modell im Sinne eines schlüssigen Gesamtkonzeptes wirksam zu gestalten.

Fazit: Sehen Sie sich die psychotherapeutischen Möglichkeiten in unterschiedlichen Settings an!

\section{Wie sollte nun konkret die Behandlung eines solchen Patienten unter diesen Ge- sichtspunkten aussehen?}

\begin{tabular}{l}
\hline Fallbeispiel \\
Ein Patient mit einer rezidivierenden depressiven Störung, gegenwärtig schwerer depressiver Episode \\
und bestehenden Suizidgedanken wird über die Notaufnahme einer psychiatrischen Klinik auf eine \\
Akutstation aufgenommen. Er ist ablehnend, verweigert jede Zusammenarbeit, schreit herum, beleidigt \\
Sie und entwertet die therapeutischen Bemühungen. Die Behandlung erfolgt zunächst nicht auf freiwil- \\
liger Basis; der Patient bagatellisiert die Schwere der depressiven Symptomatik und seine Suizidgedan- \\
ken. Sie als zuständiger ärztlicher Behandler sind zunächst gezwungen, freiheitsentziehende Maßnah- \\
men gegen den Willen des Patienten in die Wege zu leiten. Dies ist bereits ein Umstand, der für den \\
Beginn einer ambulanten Richtlinienpsychotherapie undenkbar wäre.
\end{tabular}

Trotz dieser ungünstigen Ausgangssituation besteht die Möglichkeit, eine vertrauensvolle therapeutische Beziehung aufzubauen. Lassen Sie sich trotz dieser anfänglichen Ablehnung nicht entmutigen: Nur sehr selten sind Patienten dauerhaft dranghaft suizidal!

Die folgenden Maßnahmen können helfen, einen therapeutischen Zugang zu dem Patienten $z u$ erreichen, eine tragfähige, komplementäre (d.h. auf die Bedürfnisse des Patienten orientierte) therapeutische Beziehung aufzubauen:

1. Problemanalyse und Sortierung, Zielklärung

2. Aufbau einer Therapie- ggf. sogar Veränderungsmotivation

3. Diagnostik, sowie Entwicklung eines minimalistischen Störungs- und ggf. Veränderungsmodells

4. Stabilisierung und Aufbau der Belastbarkeit

5. Vermittlung an weitere Behandlungsmöglichkeiten, zum Beispiel tagesklinische oder Rehabilitationsbehandlungen

Auch kleine Schritte sind Fortschritte - in dem geschilderten Fall ist auch der Übergang von einer nicht-freiwilligen zu einer freiwilligen Behandlung ein großer Schritt.

Für die Begleitung des Belastbarkeitsaufbaus bietet der vollstationäre Rahmen Möglichkeiten, die einem ambulanten Therapeuten nicht zur Verfügung stehen. So können die psychotherapeutischen Maßnahmen, Medikation, ergo- und physiotherapeutische Maßnahmen sowie sozialmedizinische Behandlungsanliegen und andere Rahmenbedingungen aufeinander abgestimmt werden. 
Der Beginn der nächsten Phase in Bezug auf das Fallbeispiel würde bedeuten: „Der Patient ist nicht mehr akut suizidal, erkennt, dass er unter einer Depression leidet und möchte sich freiwillig in Ihre Weiterbehandlung begeben."

\section{Wie können Sie als Behandler in dieser Phase psychotherapeutische Elemente in die Behandlung einfließen lassen?}

Durch die „freiwillige“ Behandlung kann dem Patienten ein großer Teil der initial durch den Therapeuten abgenommenen Verantwortung zurückgegeben werden, was im Idealfall eine Stärkung der Selbstwirksamkeitserwartung des Patienten über den Behandlungsverlauf bedeuten kann. Folgende Interventionen stellen eine beispielhafte Behandlung des Patienten aus dem Fallbeispiel auf einer Schwerpunktstation dar:

- Psychodiagnostik oder die Frage „Was hat der Patient neben der Depression?“

- Entwicklung eines allgemeinen, spezifischen und individuellen Störungsmodells bei genau diesem Patienten

- Zielklärung beispielsweise schriftlich festhalten und zu den Zielen passende Interventionen im Rahmen der Einzel- und Gruppenpsychotherapie-Sitzungen planen

Welche Themen könnten sich für den geschilderten Fall im Einzel- oder Gruppensetting ergeben?

Wenn Sie als Gruppentherapeut auf Ihrer Station eingesetzt werden, können Sie diesem Patienten Techniken im Umgang mit dysfunktionalen Gedanken und Veränderungsmöglichkeiten vermitteln und seine sozialen Kompetenzen steigern sowie angenehme Aktivitäten mit ihm planen.

Fragen

- Welche Tätigkeiten hat der Patient im Rahmen der depressiven Erkrankung vernachlässigt? Welche depressiven Denkmuster treten auf und welche Techniken können angewendet werden, um diese zu verändern?

- Welche zwischenmenschlichen Interaktionsprobleme haben möglicherweise zur Entwicklung der depressiven Symptomatik beigetragen oder traten erst durch die Depression auf?

Im Rahmen der Leitung der therapeutischen Gruppen haben Sie den wesentlichen Vorteil, dieses Interaktionsverhalten gewissermaßen in „Echtzeit“ zu beobachten und im Idealfall therapeutisch zu nutzen.

\section{Hilfreiche Fragen}

- Was fällt in der Gruppe besonders auf?

- Wie verhält sich der Patient als Einzelperson in der Gruppe?

- Wie wirkt der jeweilige Patient auf Sie?

- Gibt es Besonderheiten oder Regelmäßigkeiten? 
Als Einzeltherapeut könnten Sie die Interventionen in den Einzelgesprächen vertiefen sowie besondere Maßnahmen planen wie die Ehefrau zu einem Angehörigengespräch einladen, den Transfer des in der Gruppentherapie Erlernten planen oder individuelle Hindernisse besprechen.

Je nachdem wie die Fortschritte und die Belastbarkeit sowie Veränderungsmotivation des Patienten eingeschätzt werden, sollte die Therapie in einer Tagesklinik oder direkt im ambulanten Bereich erfolgen. Die vollstationäre Behandlung sollte wirklich nur so lange wie absolut notwendig dauern.

\section{Den allermeisten Patienten wird durch eine Hospitalisierung nur geschadet.}

Anhand des Fallbeispiels könnte dies so aussehen: „Der Patient hat sich weiter stabilisiert, eine tragfähige Medikation erhalten, erkennt sein Störungsbild der Depression, hat einige angenehme Aktivitäten aufgebaut und erarbeitet, dass seine zwanghaften Persönlichkeitsanteile sowie Konflikte mit der Ehefrau mit der Entwicklung der Depression zusammenhängen."

In der Tagesklinik könnten Sie diese psychotherapeutischen Elemente jetzt mit in die multimodale Therapie einfließen lassen:

- Verhaltensexperimente (bezogen auf die Reduktion der Zwanghaftigkeit)

- Vertiefung des Konfliktmanagements (bezogen auf die Beziehung zur Ehefrau; z.B. durch Rollenspiele)

- Übertragung von mehr Verantwortung für die Umsetzung des Erlernten sowie therapeutische Hausaufgaben - zur Vorbereitung der ambulanten Weiterbehandlung

Sollte eine Indikation für eine ambulante Psychotherapie bestehen, so ergeben sich die Unterschiede in der fortführenden ambulant-psychotherapeutischen Weiterbehandlung ganz automatisch; allein schon durch die formalen Gegebenheiten: Sitzungsfrequenz durchschnittlich 1 x/Woche, erhöhte Vor- und Nachbereitungszeit und inhaltlich intensivierte Arbeit.

Fazit: So haben die jeweiligen Etappen und Settings eigene Chancen und Grenzen bzw. unterschiedliche Möglichkeiten, psychotherapeutische Elemente in die Behandlung zu integrieren.

\section{Häufige Limitationen}

$\mathrm{Zu}$ den am häufigsten bestehenden limitierenden Faktoren im Aufbau einer therapeutischen Beziehung zählt die Zeit. Sie als junges ärztliches Teammitglied sind durch die vielfältigen Aufgaben auf der Station, die zu leistenden Bereitschaftsdienste und Ihre eigene Ausbildung stark in Ihren Möglichkeiten eingeschränkt. Darüber hinaus haben Sie den Spagat zwischen einem „weißkitteltragenden Schulmediziner“ und einem psychotherapeutisch tätigen Kollegen zu bewältigen.

Häufig neigt man als ärztlicher Behandler dazu, dem Patienten bestimmte Entscheidungen in eher direktivem Stil nahezulegen (zum Beispiel eine Empfehlung für eine gewisse Medikation) und ihn aufgrund vorliegender Ambivalenz mit der 
ärztlichen Übernahme einer Entscheidung zu entlasten. Dies kann in deutlichem Widerspruch zu einer aus psychotherapeutischer Sicht notwendigen Verantwortungsübernahme durch den Patienten stehen. Besondere Bedeutung für Ihre Ausbildung und die Patientenbehandlung kommt dabei einer regelmäßigen Supervision (Einzel- und Gruppensetting) zu.

Diese Supervision sollte neben fachspezifischen Aspekten auch immer die Selbstreflexion des Ausbildungskandidaten berücksichtigen. Darüber hinaus sollte die Fallbesprechung auch in einer Gruppensupervision, welche durch einen erfahrenen Therapeuten geleitet wird, fortgesetzt werden. In vielen Ausbildungsinstituten und Kliniken hat sich in der Nachfolge zur „Balintgruppe“ auch die Interaktionelle Fallarbeit (IFA) als Möglichkeit der Gruppensupervision entwickelt. Idealerweise sollte die Leitung dieser Gruppen nicht ausschließlich durch ärztliche Kollegen, sondern auch durch psychologische Kollegen und eventuell sogar therapieschulenübergreifend erfolgen.

Fazit: Machen Sie nicht den Fehler vieler Ihrer ärztlichen Kollegen, schon allein aufgrund des Arztseins selbst alles hinbekommen zu wollen. Supervision steht Ihnen zu und Sie sollten sich diese auch einfordern. Sie ist eine Notwendigkeit, Chance und stetiger Begleiter in guten wie in schlechten Tagen (Sitzungen)! 